\title{
The use of nomograms in LDR-HDR prostate brachytherapy
}

\author{
Ma Carmen Pujades, MScl, Cristina Camacho, ScD!, Jose Perez-Calatayud, PhDI.2, José Richart, MSc2, Jose Gimeno, ScD3. \\ Françoise Lliso, ScD!, Vicente Carmona, ScD!, Facundo Ballester, PhD4, Vicente Crispín, ScD33, Silvia Rodríguez, PhD2, \\ Alejandro Tormo, PhDl \\ IRadiotherapy Department, La Fe University Hospital, Valencia, Spain, 2Radiotherapy Department, Hospital Clínica Benidorm, Benidorm, \\ Spain, ${ }^{3}$ Medical Physics Department, Fundación Instituto Valenciano de Oncología (IVO), Valencia, Spain, ${ }^{4}$ Atomic, Molecular and Nuclear \\ Department, University of Valencia, Valencia, Spain
}

\begin{abstract}
Purpose: The common use of nomograms in Low Dose Rate (LDR) permanent prostate brachytherapy (BT) allows to estimate the number of seeds required for an implant. Independent dosimetry verification is recommended for each clinical dosimetry in BT. Also, nomograms can be useful for dose calculation quality assurance and they could be adapted to High Dose Rate (HDR). This work sets nomograms for LDR and HDR prostate-BT implants, which are applied to three different institutions that use different implant techniques.

Material and methods: Patients treated throughout 2010 till April 2011 were considered for this study. This example was chosen to be the representative of the latest implant techniques and to ensure consistency in the planning. A sufficient number of cases for both BT modalities, prescription dose and different work methodology (depending on the institution) were taken into account. The specific nomograms were built using the correlation between the prostate volume and some characteristic parameters of each BT modality, such as the source Air Kerma Strength, number of implanted seeds in LDR or total radiation time in HDR.

Results: For each institution and BT modality, nomograms normalized to the prescribed dose were obtained and fitted to a linear function. The parameters of the adjustment show a good agreement between data and the fitting. It should be noted that for each institution these linear function parameters are different, indicating that each centre should construct its own nomograms.

Conclusions: Nomograms for LDR and HDR prostate brachytherapy are simple quality assurance tools, specific for each institution. Nevertheless, their use should be complementary to the necessary independent verification.

Key words: nomograms, prostate brachytherapy, quality assurance, independent verification.

\section{Purpose}

In the intraoperative planning of Low Dose Rate (LDR) interstitial permanent prostate brachytherapy (BT), the use of nomograms (a two-dimensionally plotted function with $n$ parameters, from which, knowing $n-1$ parameters, the unknown one can be read) is very common. Their use allows the estimation of the number of seeds required for an implant according to the prostate volume [1-3]. The physical origin of nomograms in permanent prostate brachytherapy is due to the correlation between dose and target volume [4]. There are recommendations indicating the need for carrying out an independent check of each clinical patient dosimetry calculation in BT [5]. To introduce these recommendations, some methods which are able to perform this brachytherapy dosimetry verification in an accurate and simple way, were proposed. A technique recently developed by Carmona et al. [6], suitable for both LDR and High Dose Rate (HDR) prostate brachytherapy was among these methods. Similarly, the nomogram can be useful to determine the necessary number of seeds and as a dose calculation quality assurance tool [7] and for that reason, being a suitable complement to independent calculation. In the treatment of intermediate and high risk prostate cancer groups, HDR techniques are being established as a common complement to the external radiotherapy (ERT). This is mainly due to their radiobiological advantages, proper clinical target volume coverage and well optimized dose with respect to organs at risk. In this brachytherapy modality, the need for estimating in advance total Air Kerma Strength does not exist. The parameter that is equivalent to the seed activity in LDR in the construction of a nomogram turns to be the product of the total radiation time $(T)$ and source Air Kerma Strength $\left(S_{K}\right)$. Therefore, similarly to LDR im- 
plants, a correlation between $S_{K}$ at the particular treatment time of the application and the prostate volume may be carried out. Once more, that relationship would be useful as a quality control in HDR treatments in addition to the necessary independent dosimetry verification, using, for example, the methods discussed by Carmona et al. [6]. The aim of this work was to set specific nomograms for both, LDR and HDR prostate brachytherapy implants for different dose prescriptions. Nomograms from three institutions that use different implant techniques were compared.

\section{Material and methods}

The suitable parameters to set LDR and HDR prostate brachytherapy implants nomograms from three different institutions were studied. LDR-BT was performed since 2004, and HDR-BT since 2009 in the institutions part of this study, using different implant techniques and methodologies. Patients throughout 2010 till present (April 2011) were considered for this study to be the representative of the latest implant techniques, as well as to ensure consistency and experience in the planning methodology. The number of patients considered by modality and institution is distributed as follows:

Institution A:

LDR: 148 implants. 69\% of them were exclusive (160 Gy), $23 \%$ were boost (108 Gy) after ERT and $8 \%$ were salvage cases (120 Gy).

HDR: 54 applications. In 54\% of them the prescribed dose was $15 \mathrm{~Gy}$, and $9 \mathrm{~Gy}$ in the $37 \%$. Dose prescription in the remaining cases was found in the range $10 \mathrm{~Gy}<D<15 \mathrm{~Gy}$. Institution B:

LDR: 52 implants, $46 \%$ of them, were exclusive (160 Gy), $48 \%$ were boost (108 Gy) after ERT and the remaining $6 \%$ were salvage cases (120 Gy).

HDR: 73 applications. In $86 \%$ of them the prescribed dose was $9.5 \mathrm{~Gy}$ and the rest of cases were found in the range 10 Gy $<D<15$ Gy.

\section{Institution $\mathrm{C}$ :}

LDR: 123 implants, $70 \%$ of them were exclusive (145 Gy), $26 \%$ were boost (108 Gy) after ERT and the remaining $4 \%$ were salvage cases (130 Gy).

As it could be seen, different doses were prescribed within the same institution and work methodology, according to each specific case. The implant was done using the guidance of a biplane ultrasound rectal probe for the anatomical data acquisition, and performing the implant in real time. According to the considered institution, the techniques used in each modality (LDR or HDR) were different:

a) LDR modality:

The sources used for permanent implants were: ${ }^{125} \mathrm{I}$ IsoSeed seeds (IBT Bebig Group), either linked by IsoCord sources trains - in which the seeds remained spaced $1 \mathrm{~cm}$ (institution A), or loosed seeds used with the afterloader SeedSelectron (Nucletron) system, which allowed the possibility of implanting several seeds together (institution B); and the STM1251 125I seeds (Bard Medical) linked by "SourceLink" trains in which the seeds centres could be separated by multiples of $0.5 \mathrm{~cm}$ placing spacers between them (institution C).
For the calculation of dose distribution in LDR several treatment planning systems were used depending on the institution: the SPOT-PRO software v3.1 (Nucletron BV, Veenendal, the Netherlands) in institution A and B, and the VariSeed software v8.0 (Varian Medical Systems, Palo Alto, CA), in institution C. Plans were generated in order to obtain the appropriate dose coverage of the PTV, according to the case: 145-160 Gy, 120-130 Gy or 108 Gy in exclusive implants, salvage cases or boost, respectively. To calculate LDR nomograms, the correlation between prostate volume and the total activity of implanted seeds was evaluated.

\section{b) HDR modality:}

The ${ }^{192}$ Ir mHDR-v2 source and the HDR MicroSelectron afterloader device (Nucletron BV, Veenendal, the Netherlands) were applied in both, institution A and B. Both institutions used the Oncentra Prostate software (v3.1.11, Nucletron BV, Veenendal, the Netherlands) for treatment planning and dose calculation. In order to acquire similar relationship to the one obtained in the LDR modality, the possible correlation between the target volume and the characteristic parameters of the HDR application was studied for the nomogram construction.

\section{Results}

\section{a) LDR modality:}

It was found that the product of the total number of implanted seeds and the $S_{K}$ average value, normalized to the prescribed dose, follows linear relation with the target volume. The linear function is expressed as follows:

$$
\frac{N_{\text {seeds }} \times S_{K}}{\mathrm{D}}=a \times V+b
$$

where $N_{\text {seeds }}$ is the number of implanted seeds, $S_{K}$ is the Air Kerma Strength of the sources $\left(\mu \mathrm{Gy} \times \mathrm{m}^{2} \times \mathrm{h}^{-1}\right), D$ is the prescribed dose (Gy) and $V$ is the prostate volume $\left(\mathrm{cm}^{3}\right)$. The graphical representations of expression (1) for each institution are shown in Figs. 1, 2 and 3, constituting the specific nomograms for LDR modality. Linear fit parameters ( $a$ and $b$ ) and the linear correlation coefficient for each fit are presented in Table 1. It can be seen, that similar expressions work properly for cases with different prescription dose $\left(R^{2}\right.$ coefficients ranging from $\left.0.851-0.926\right)$. It is also observed that $a$ and $b$ vary between institutions due to differences in methodology and technique. For example, for similar prostate volume and prescribed dose, the higher value of the parameter $a$ in the institution B nomogram would indicate that in this institution the total $S_{K}$ of implanted ${ }^{125}$ I seeds was higher than in the other two institutions.

\section{b) HDR modality:}

In order to acquire similar nomograms than those obtained in LDR modality, an appropriate relationship between the characteristic parameters of a HDR application and the prostate volume was found. The product of the total radiation time and the source $S_{K}$ at the time of HDR application, normalized to the prescribed dose, fits to a linear function with the target volume. Here, the total number of 


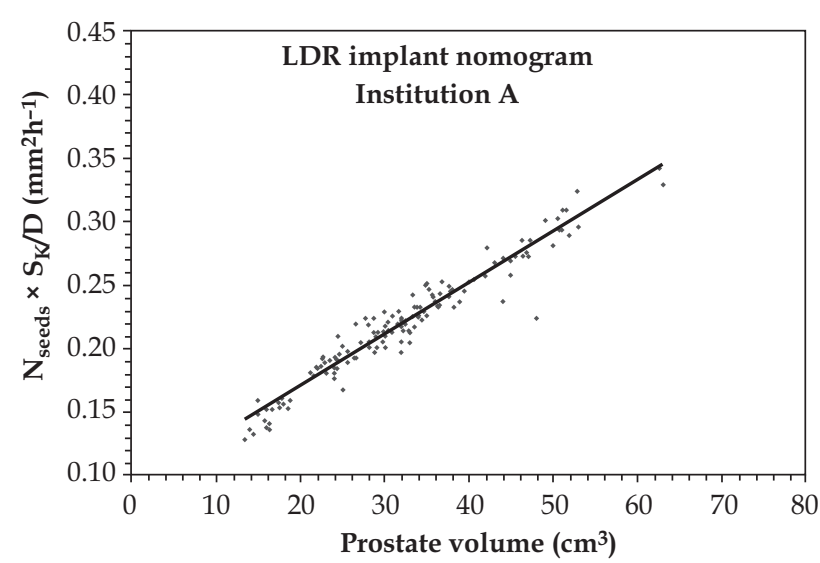

Fig. 1. Institution A LDR-BT nomogram. Data was fitted to a linear expression (equation [1])

implanted seeds was replaced with the total radiation time. The linear function is expressed as follows:

$$
\frac{T \times S_{K}}{\mathrm{D}}=a^{\prime} \times V+b^{\prime}
$$

where $S_{K}$ is the source Air Kerma Strength $\left(\mu \mathrm{Gy} \times \mathrm{m}^{2} \times \mathrm{h}^{-1}\right)$, $T$ is total radiation time (h), $D$ is the prescribed dose (cGy), and $V$ is the target volume $\left(\mathrm{cm}^{3}\right)$. Similarly to the LDR modality, fitted parameters $a^{\prime}$ and $b^{\prime}$, and linear correlation coefficient were obtained. The results are shown in Figs. 4 and 5 (for institutions A and B, respectively), and Table 2. It has been demonstrated, that the linear fit was suitable in both cases $\left(R^{2}\right.$ of 0.940 and 0.851 for institutions $A$ and $B$, respectively). In this case, the values of the parameters $a^{\prime}$ and $b^{\prime}$ do not differ so much, as compared with the LDR modality case, suggesting that both institutions follow similar methodology when performing HDR planning. It was also found, that the institution B accepts higher overdose volumes, since for a given prostate volume and applied dose, the $a^{\prime}$ parameter of the nomogram was slightly higher than that in institution A.

\section{Discussion}

Not only the nomogram provides an estimation of the quantity of necessary seeds in LDR implants within a certain range, but a simple additional quality assurance tool

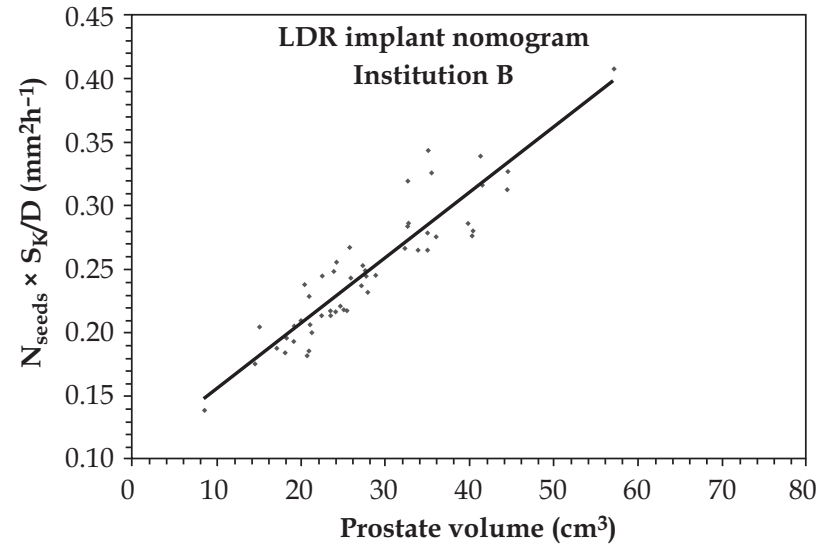

Fig. 2. Institution B LDR-BT nomogram. Data was fitted to a linear expression (equation [1])

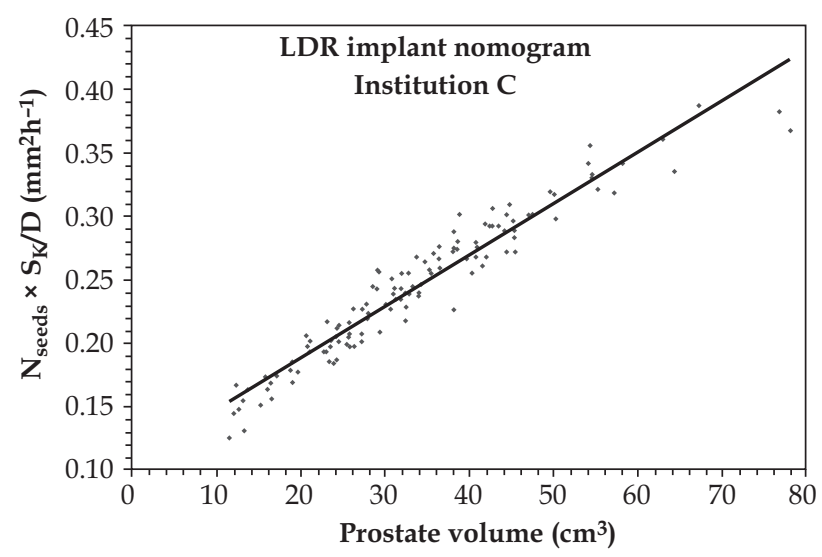

Fig. 3. Institution C LDR-BT nomogram. Data was fitted to a linear expression (equation [1])

that could be applied to both LDR and HDR. At the end of our clinical dosimetry procedure in prostate brachytherapy, and always before the treatment, it is established that in addition to the independent verification (according to the Carmona et al. method [6]), the value predicted by the nomogram is contrasted with the value obtained in dosimetry. This check is done by a quick calculation using fitting expressions (1) and (2). However, it is important to emphasize that the nomograms should not substitute an independent verification. The main reason for that is the lack of

Table 1. Parameters obtained from the fit of Figures 1, 2 and 3 curves to a linear expression (equation [1])

\begin{tabular}{|c|c|c|c|}
\hline BT modality & Institution & Linear fit $\frac{N_{\text {seeds }} \times S_{k}}{D}=a \times V+b$ & $R^{2}$ \\
\hline & A & $\begin{array}{l}a=(0.404 \pm 0.008) \times 10^{-4}\left(\mathrm{~cm}^{-1} \mathrm{~h}^{-1}\right) \\
b=(9.0 \pm 0.3) \times 10^{-4}\left(\mathrm{~cm}^{2} \mathrm{~h}^{-1}\right)\end{array}$ & 0.940 \\
\hline \multirow[t]{2}{*}{ LDR } & B & $\begin{array}{l}a=(0.52 \pm 0.03) \times 10^{-4}\left(\mathrm{~cm}^{-1} \mathrm{~h}^{-1}\right) \\
b=(10.4 \pm 0.9) \times 10^{-4}\left(\mathrm{~cm}^{2} \mathrm{~h}^{-1}\right)\end{array}$ & 0.851 \\
\hline & C & $\begin{array}{l}a=(0.407 \pm 0.010) \times 10^{-4}\left(\mathrm{~cm}^{-1} \mathrm{~h}^{-1}\right) \\
b=(10.5 \pm 0.4) \times 10^{-4}\left(\mathrm{~cm}^{2} \mathrm{~h}^{-1}\right)\end{array}$ & 0.926 \\
\hline
\end{tabular}




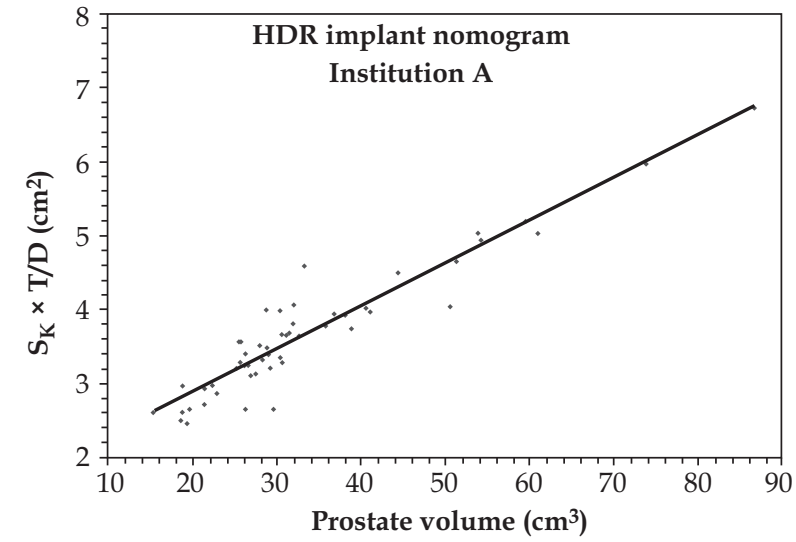

Fig. 4. Institution A HDR-BT proposed nomogram. Data was fitted to a linear expression (equation [2])

Table 2. Parameters obtained from the fit of Figures 4 and 5 curves to a linear expression (equation [2])

\begin{tabular}{lccc} 
BT modality Institution & $\begin{array}{l}\text { Linear fit } \\
\frac{T \times S_{k}}{D}=a^{\prime} \times V+b^{\prime}\end{array}$ & \\
& & $R^{2}$ \\
\hline ADR & $a^{\prime}=(5.8 \pm 0.8) \times 10^{-2} \mathrm{~cm}^{-1}$ & 0.888 \\
& & $b^{\prime}=(1.7 \pm 0.3) \mathrm{cm}^{2}$ & \\
\cline { 2 - 3 } & B & $a^{\prime}=(6.3 \pm 0.5) \times 10^{-2} \mathrm{~cm}^{-1}$ & 0.940 \\
& $b^{\prime}=(1.70 \pm 0.14) \mathrm{cm}^{2}$ &
\end{tabular}

information about the distribution of absorbed dose within the implant volume. Nomograms check the consistency in the methodology of work.

This paper presents nomograms from diverse institutions which use different methodologies and techniques. We conclude that for all of them a linear data fit is adequate. A significant result is that nomograms for both BT modalities were normalized to the prescribed dose. It means that there is no need to construct a separate nomogram for each prescription dose, as it was in the standard procedure. It should also be noted that, although appropriate fits were obtained in these three institutions nomograms, their nomograms differ, as proved by the different values obtained for the fitting parameters, indicating that each nomogram is characteristic for each institution. Different institutions may have different philosophies when performing planning. This study highlights the importance of each institution to obtain their own linear fitted parameters, if nomograms are going to be applied in specific clinical routine.

\section{Conclusions}

Nomograms for LDR and those proposed for HDR brachytherapy modalities were obtained, being an additional and simple quality assurance tools, which vary according to the methodology and technique, being specific for each institution.

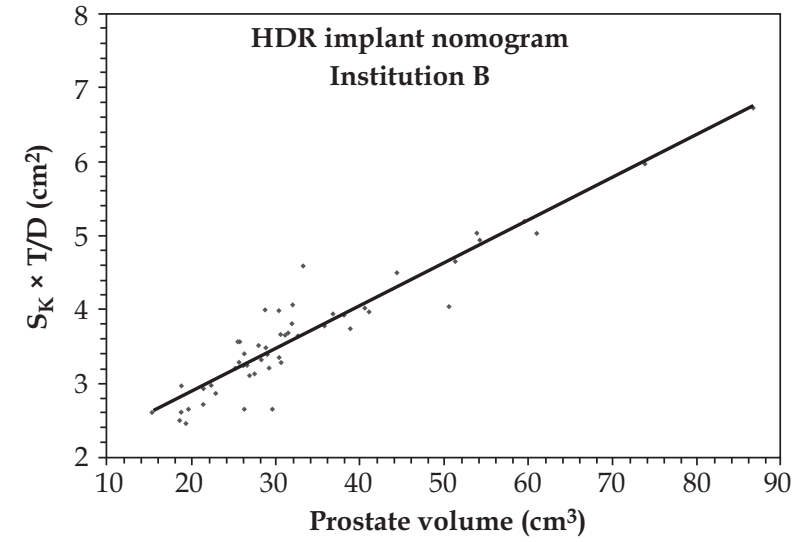

Fig. 5. Institution B HDR-BT proposed nomogram. Data was fitted to a linear expression (equation [2])

\section{Acknowledgement}

This study was supported in part by Nucletron, B.V. (The Netherlands); Generalitat Valenciana (Project PROMETEO2008/114); and Ministerio de Ciencia e Innovación, Spain (Project No. FIS2010-17007).

\section{References}

1. Aronowitz JN, Karolczuk K. The need for institution-specific nomograms for the preoperative prediction of seed requirements when intraoperative planning is utilized for LDR prostate brachytherapy. Brachytherapy 2008; 7: 131-132.

2. Cui Y, Doyle L, Podder T et al. Can the number of Leftover Seeds be reduced? A study of prostate volume, nomogram and delivered activity for ultrasound-guided 125I prostate seed implant. Brachytherapy 2011; 10: 86.

3. Al-Qaisieh B, Brearley E, St Clair S et al. A study of a pre-treatment method to predict the number of I- 125 seeds required for prostate brachytherapy. Int J Rad Oncol Biol Phys 2006; 65: 304-307.

4. Li T, Fountain BL, Duffy EW. Physical derivation of nomograms in permanent prostate. Brachytherapy 2010; 9: 50-54.

5. A practical guide to quality control of brachytherapy equipment. European guidelines for quality assurance radiotherapy. EN: Jack Venselaar and Jose Perez-Calatayud editors. ESTRO Booklet No. 8. 2004.

6. Carmona V, Perez-Calatayud J, Lliso F et al. A program for the independent verification of brachytherapy planning system calculations. J Contemp Brachyther 2010; 2: 129-133.

7. Nag S, Ciezki JP, Cormack R et al. Intraoperative planning and evaluation of permanent prostate brachytherapy: Report of the American Brachytherapy Society. Int J Rad Oncol Biol Phys 2001; 51: $1422-1430$ 